\title{
TK1 Membrane Expression May Play a Role in the Invasion Potential of A549 Lung Cancer Cells
}

\author{
Evita G. Weagel ${ }^{1 *}$, Juan Mejía ${ }^{2}$, Roman Kovtunn', Joshua Keller ${ }^{1}$, \\ Juan A. Arroyo ${ }^{2}$, Richard A. Robison' ${ }^{1}$, Kim 0'Neill ${ }^{1}$ \\ ${ }^{1}$ Department of Microbiology and Molecular Biology, Brigham Young University, Provo, UT, USA \\ ${ }^{2}$ Department of Physiology and Developmental Biology, Brigham Young University, Provo, UT, USA \\ Email: *evitaweagel@gmail.com
}

How to cite this paper: Weagel, E.G., Mejía, J., Kovtun, R., Keller, J., Arroyo, J.A., Robison, R.A. and O'Neill, K. (2018) TK1 Membrane Expression May Play a Role in the Invasion Potential of A549 Lung Cancer Cells. Journal of Cancer Therapy, 9, 605-612.

https://doi.org/10.4236/jct.2018.98052

Received: June 20, 2018

Accepted: August 21, 2018

Published: August 24, 2018

Copyright $\odot 2018$ by authors and Scientific Research Publishing Inc. This work is licensed under the Creative Commons Attribution International License (CC BY 4.0).

http://creativecommons.org/licenses/by/4.0/

\begin{abstract}
Thymidine kinase 1 (TK1) is a well-studied cancer biomarker. It is commonly found upregulated in the serum of cancer patients, and its levels correlate with stage and grade, disease progression, and prognosis. It has recently been reported that TK1 localizes on the plasma cell membrane of hematological and solid malignancies, and not on the membrane of normal healthy cells, and while on the membrane, TK1 has enzymatic activity. However, the function of TK1 on the surface membrane is not well understood. Here, we hypothesize that it may have a role in tumor invasion and migration. It has been shown that TK1 expression levels positively correlate with epithelia to mesenchymal transition (EMT) markers in patients with breast cancer as they progress from HER2+ to triple negative breast cancer. In this study, we silenced TK1 expression by siRNA and show that TK1's membrane expression is significantly downregulated at 60 hours post transfection. Using a Matrigel-based quantitative invasion assay, we measured cell invasion potential in cells either expressing or lacking TK1 on their membrane and found that cells that lack TK1 on their membrane exhibit decreased invasion potential. These results suggest that TK1's presence on the membrane may play a role in invasion and cell migration in cancer.
\end{abstract}

\section{Keywords}

Thymidine Kinase 1, Invasion, EMT

\section{Introduction}

Thymidine kinase 1 (TK1) is a nucleotide salvage pathway enzyme primarily re- 
sponsible for converting deoxythymidine to deoxythymidine monophosphate, and upon subsequent phosphorylation is incorporated into DNA [1]. During normal cell proliferation, TK1 is usually found in the cytoplasm where its expression is cell cycle-dependent, peaking during G1/S phase [2]. TK1 levels can also increase in response to DNA damage, especially after chemotherapy and radiotherapy. This increase is thought to provide cancer cells with a supply of nucleotides for DNA repair rather than for proliferation, and serves as a support mechanism to promote the survival of cancer cells [3]. Due to its tight regulation by the cell cycle, TK1 has been extensively studied in the context of cancer diagnostic biomarkers, where it has been shown to be upregulated in tissue and serum in both solid tumors and hematological malignances. TK1's prognostic and diagnostic potential has been demonstrated using the traditional TK activity radioassay for hematological malignancies and solid tumors including lung, breast, colon, bladder, cervical, and colorectal [4]-[9]. TK1 levels in serum have also been shown to have diagnostic potential in bladder cancer, cervical carcinoma, gastric cancer, non-small cell lung cancer, colorectal cancer, and renal cell carcinoma. In summary, high TK1 serum levels correlate with tumor aggressiveness and can be indicative of early events in carcinogenesis [10] [11]. While TK1 has mostly been studied as a cancer biomarker in the serum, we have recently shown that TK1 localizes on the plasma membrane of cells, this is an event independent of proliferation and appears to be exclusive to malignant cells. For these reasons, TK1 may be a novel target for cancer immunotherapy. However, understanding TK1's function on the membrane is important as we approach the development of immunotherapy using TK1 as a target. Recently, it was reported that TK1 expression levels correlate with EMT markers in breast cancer in clinical samples as the disease progresses to triple negative breast cancer [12]. Additionally, because serum TK1 correlates with disease progression and aggressiveness, we hypothesized that membrane TK1 could have an immunoregulatory role and could aid in the invasion potential of cancer cells. In this study, we found that when membrane TK1 expression is silenced, cells lose invasion potential. These results suggest that TK1 may have a role in migration and immunoregulation, as well as in epithelial mesenchymal transitions (EMT).

\section{Materials and Methods}

\subsection{Cell Line and Cell Culture}

The non-small cell lung carcinoma A549 cell line was purchased from ATCC (Catalog \# CCL-185 $5^{\mathrm{mm}}$ ) and cultured in RPMI 1640 medium supplemented with $10 \%$ fetal bovine serum (FBS) and $2 \mathrm{mM}$ L-glutamine (both from Thermo Fisher Scientific, Waltham, MA). Cells were grown at $37^{\circ} \mathrm{C}$ and $5 \% \mathrm{CO}_{2}$ and fed $2-3$ times per week by removing the medium, rinsing the cells with Dulbecco's Phosphate-Buffered Saline (DPBS, Gibco Thermo Fisher Scientific, Waltham, MA), incubating them with Accutase (Stem Cell Technology, Vancouver, Canada) for detachment and subculturing in a 1:4 ratio. For this experiment, cells 
were kept at a low passage to ensure best transfection efficiency.

\section{2. siRNA Library}

Three validated siRNA against TK1 and control siRNA (GAPDH) were purchased from Thermo Fisher Scientific (catalog \# 4390824, siRNA IDs s14158, s14159, and s14160 for TK1, and catalog \# 4390849 for GAPDH). siRNA was resuspended in nuclease-free water at $50 \mathrm{uM}$, aliquoted, and stored at $-80^{\circ} \mathrm{C}$. For working siRNA concentrations, siRNA was diluted to $10 \mathrm{uM}$ in nuclease-free water.

\subsection{Transfection}

Two hundred-thousand A549 cells were plated in 6 well-plates overnight to allow for attachment, after which, the medium was removed, and cells were washed with DPBS and $2 \mathrm{~mL}$ serum-free RPMI 1640 was added to each well. Lipid-nucleic acid complexes of RNAiMAX (Thermo Fisher Scientific, Waltham, MA, catalog \# 13778030) and siRNA were prepared according to the manufacturer's instructions and added to the cells. 12 hours after transfection, medium and lipid-nucleic acid complexes were removed from cells and fresh complete RPMI 1640 medium was added to the cells. Cells were used at 60 hours post transfection, as recommended by manufacturer. (Cells treated with siRNA ID 14158 were named siRNA 58, those treated with siRNA ID 14159 were named siRNA 59, and those treated with siRNA ID 14160 were named siRNA 60).

\subsection{Flow Cytometry}

Transfected A549 cells were rinsed with DPBS, treated with Accutase at $37^{\circ} \mathrm{C}$ for 5 - 10 mins to allow for detachment, and then rinsed with complete medium. Cells were pelleted and resuspended at $1 \times 10^{6} \mathrm{cells} / \mathrm{mL}$ in Cell Staining Buffer (BioLegend, San Diego, CA) and $200 \mathrm{uL}$ of cells were placed in individual microcentrifuge tubes and incubated with $5 \mathrm{uL}$ of Fc block (Human TruStain FcX ${ }^{\mathrm{mw}}$, BioLegend, San Diego, CA) for 10 minutes at room temperature, followed by incubation with 1 ug of anti-TK1 antibody (ab91651, Abcam, Cambridge, United Kingdom) for 15 minutes on ice. Cells were then washed with 1 mLCell Staining Buffer, incubated with anti-rabbit AlexaFluor647 antibody (Thermo Fisher, Waltham, MA, cat \# A27040) and incubated on ice in the dark for 15 minutes. Cells were washed 2X with Cell Staining Buffer and resuspended in Fluorescence-activated cell sorting (FACS) buffer. FACS buffer was made with phosphate-buffered saline (PBS), 2\% calf serum (Thermo Fisher, Waltham, MA), 1 mM EDTA (Thermo Fisher, Waltham, MA, CAS 6381-92-6), and 0.1\% sodium azide (Sigma Aldrich, St. Louis, MO, CAS 26628-22-8). We collected $1 \times$ $10^{4}$ events per sample in a flow cytometer (Accuri C6 Plus, BD Biosicences, San Jose, CA) and data was analyzed using FlowJo software (FlowJo, Ashland, OR).

\subsection{Invasion Assay}

An xCELLigence RTCA DP instrument (Cat. 05469759001) was utilized to de- 
termine real time invasion and cell motility of A549 cells either expressing TK1 on their surface or after TK1 silencing. Invasion was assessed in 16 well CIM-Plates (Cat. 05665817001) $(n=4)$ composed of an upper and lower chamber, each containing 16 wells. The top wells were coated with a 1:40 Matrigel concentration and incubated for $4 \mathrm{hrs}$. Cells were plated in the top chamber at a concentration of 50,000 cells/well in serum-free RPMI in a total volume of 100 $\mu \mathrm{L}$ in the presence of the cells' corresponding media. The cells were allowed to migrate toward the bottom chamber wells which were filled with $160 \mu \mathrm{L}$ of $10 \%$ FBS growth medium, as a source of chemoattractant (directional migration). The cells were then placed in the RTCA DP instrument and invasion readings were taken every 5 minutes for $24 \mathrm{hrs}$.

\subsection{Statistical Analysis}

The flow cytometry data was analyzed with a one-way ANOVA test using the Sidak's correction for multiple comparisons to compare the expression of TK1 on the surface of the siRNA treated cells vs. isotype control. P-value significance was set at $\leq 0.05$. Error bars represent the standard error of the mean. Statistical analyses were performed using Prism software.

\section{Results}

\subsection{Flow Cytometry}

To assess TK1 silencing on the surface of A549 cells, a cell line known to express high levels of membrane TK1, we stained the control and siRNA treated cells with antibodies against TK1. We chose to quantify silencing by flow cytometry because TK1 is a known cytoplasmic protein and we wanted to directly assess its expression on the cell membrane only. Flow cytometry revealed that A549 cells treated with siRNA targeting TK1 displayed decreased expression of TK1 on the surface. Cells treated with siRNA 58 and siRNA 59 showed significant reduction of TK1 on the surface, while siRNA 60 did not show a significant reduction (Figure 1). Cells treated with siRNA 58 had the most dramatic silencing in TK1 surface expression with a $93.82 \%$ reduction in surface expression compared to cells treated with control siRNA. Cells treated with siRNA 59 showed a $15.98 \%$ reduction at 60 hours post transfection.

\subsection{Invasion Study}

To compare the invasion potential of A549 cells, with and without TK1 surface expression in vitro, we performed an invasion assay with A549 cells treated with control siRNA, siRNA 58, siRNA 59, and siRNA 60. In our study, the invasion potential of cells was measured by the cells crossing a layer of Matrigel from serum-free medium towards complete medium, as they seek nutrients. We performed the experiment in an xCELLigence RTCA DP instrument, which is used to determine real time invasion over a period of time. In our results, we observed that when TK1 is highly silenced on the membrane, the 

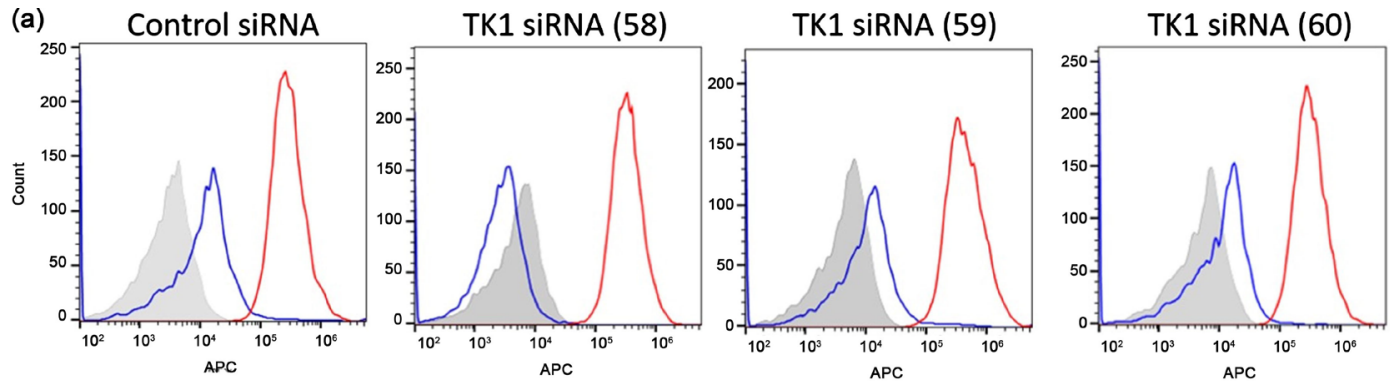

Isotype control

(b)

A549 cells- TK1 silencing all factors

(c)

A549 cells- TK1 silencing
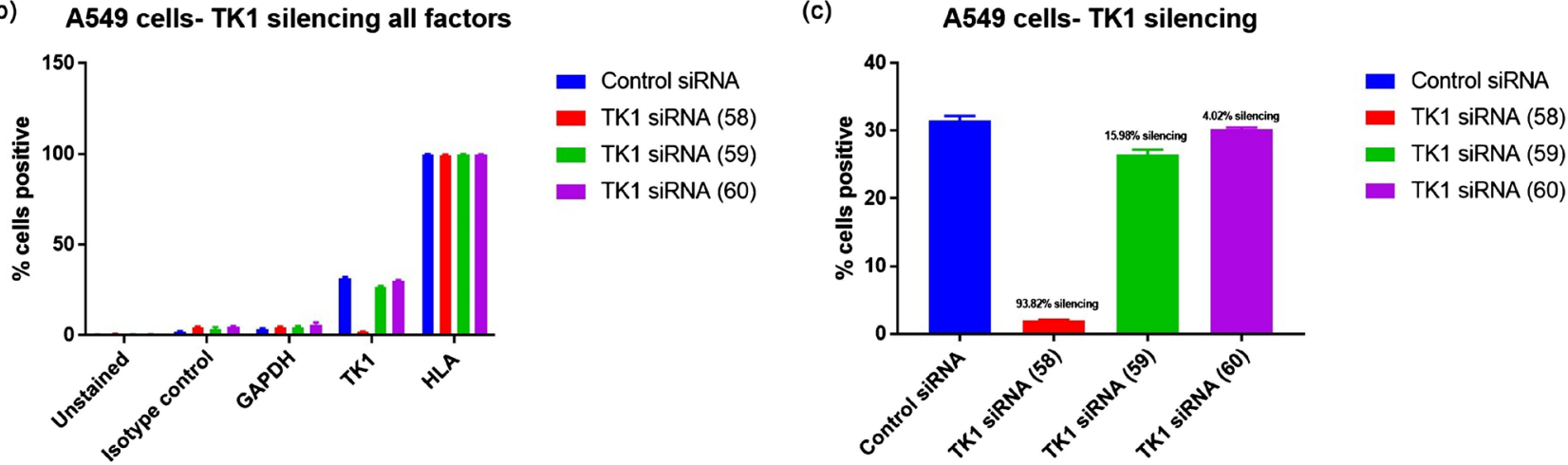

Figure 1. Membrane expression of TK1 by flow cytometry at 60 hours post siRNA transfection. (a) Histograms showing membrane expression of TK1 compared to GAPDH and HLA. A549 cells transfected with siRNA 58 show a significant shift to the left when stained with TK1 antibody, suggesting decreased TK1 expression on the surface; (b) Quantification of flow cytometry data showing the percentage of cells positive for APC stain; (c) Quantification of flow cytometry data showing that transfection of A549 cells with siRNA 58 decreases TK1 expression by $93.82 \%$.

invasion potential is reduced. In fact, only cells treated with siRNA 58, which had the most reduction of membrane TK1 expression, showed a significant reduction in invasion potential. Cells treated with siRNA 59 and siRNA 60 were statistically equally invasive to cells treated with control siRNA (Figure 2). We observed no change in cell viability before the invasion assay. Due to the short window of time post transfection, we did not evaluate growth rates. However, cell counts were similar between all transfected samples, suggesting no change in proliferation rates.

\section{Discussion}

TK1 has been studied as a serum biomarker since the late 80 's. While its clinical application has been proven to be useful as a monitoring tool for disease progression and prognosis of most cancers, our group has found it to be a potential target in the clinic. TK1 was recently reported to be differentially expressed on the membrane of hematological and solid malignancies compared to normal cells. The localization of TK1 on the membrane appears to be an event unique in malignancy, as highly proliferative tissue like colon or normal lymphocytes induced to divide do not express TK1 on their surface. This posed the question as to why malignant cells upregulate TK1 on their surface and whether this gives the malignant cells an advantage. Recently, it was reported that stemness and EMT markers were more positively and strongly correlated to TK1 levels in 


\section{A549 cell invasion}

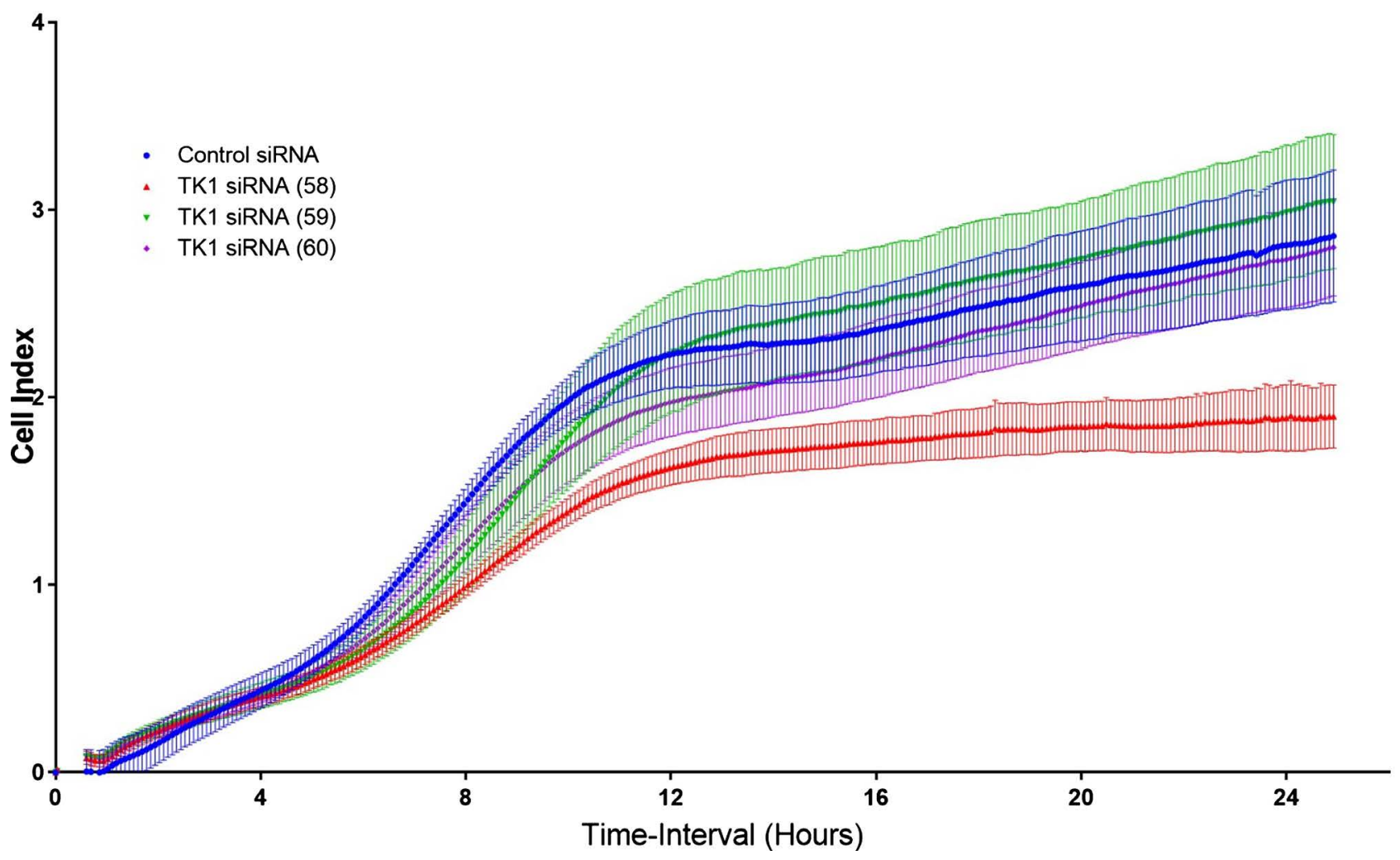

Figure 2. Invasion assay with A549 cells transfected with TK1 siRNA. Only A549 cells that had a drastic TK1 silencing show decreased invasion compared to control siRNA treated cells.

Table 1. Summary of Spearman's correlation coefficients between TK1 and cell stemness or EMT markers in HER2+ and TNBC patients.

\begin{tabular}{cccc}
\hline Gene name & Function & $\begin{array}{c}\text { HER2+: TK1 vs. gene } \\
\text { (Spearman's correlation } \\
\text { coefficient) }\end{array}$ & $\begin{array}{c}\text { TNBC: TK vs. gene } \\
\text { (Spearman's correlation } \\
\text { coefficient) }\end{array}$ \\
\hline CD44 & Cell-cell interactions, cell adhesion and migration. Stemness marker. & 0.14 & 0.24 \\
SNAI1 & Induction of epithelial to mesenchymal transition. & -0.01 & 0.13 \\
SNAI2 & Induction of epithelial to mesenchymal transition. & -0.3 & -0.14 \\
TWIST1 & Induction of epithelial to mesenchymal transition. & -0.34 & -0.02 \\
ZEB1 & Induction of epithelial to mesenchymal transition. & -0.36 & -0.35 \\
TGFB & Induction of cell migration and epithelial to mesenchymal transition. & -0.23 & -0.13 \\
\hline
\end{tabular}

triple negative breast cancer compared to HER2+ breast cancer, indicating that TK1 expression levels may also correlate with tumor invasion potential (summarized in Table 1) [12]. While not all genes show positive correlations, there is a positive trend in the Spearman's correlation coefficients between TK1 expression and these cell stemness and EMT markers between HER2+ and TNBC patients. Here we report that when we silence TK1 on the surface of A549 cells, their invasion potential decreases significantly. Further studies showing a connection of TK1 and migration or EMT markers could elucidate a potential immunoregulato- 
ry function.

\section{Conflicts of Interest}

The authors declare no conflicts of interest regarding the publication of this paper.

\section{References}

[1] Chen, Y.-L., Eriksson, S. and Chang, Z.-F. (2010) Regulation and Functional Contribution of Thymidine Kinase 1 in Repair of DNA Damage. The Journal of Biological Chemistry, 285, 27327-27335.

http://www.jbc.org/content/285/35/27327.abstract https://doi.org/10.1074/jbc.M110.137042

[2] Mutahir, Z., Clausen, A.R., Andersson, K.-M., Wisen, S.M., Munch-Petersen, B. and Piškur, J. (2013) Thymidine Kinase 1 Regulatory Fine-Tuning Through Tetramer Formation. The FEBS Journal, 280, 1531-1541.

http://www.ncbi.nlm.nih.gov/pubmed/23351158

https://doi.org/10.1111/febs.12154

[3] Alegre, M.M., Robison, R.A. and O’Neill, K.L. (2014) The Clinical Significance and Biology of Thymidine Kinase 1. Oncology-Theory and Practice, iConcept Press Ltd., Australia.

[4] Alegre, M.M., Robison, R.A. and O’Neill, K.L. (2013) Thymidine Kinase 1: A Universal Marker for Cancer. Cancer and Clinical Oncology, 2, 159.

http://www.ccsenet.org/journal/index.php/cco/article/view/26281

https://doi.org/10.5539/cco.v2n1p159

[5] Wu, B.-J., Li, W.-P., Qian, C., Ding, W., Zhou, Z.-W. and Jiang, H. (2013) Increased Serum Level of Thymidine Kinase 1 Correlates with Metastatic Site in Patients with Malignant Melanoma. Tumor Biology, 34, 643-648.

http://www.ncbi.nlm.nih.gov/pubmed/23179401 https://doi.org/10.1007/s13277-012-0591-0

[6] Fujiwaki, R., Hata, K., Moriyama, M., Iwanari, O., Katabuchi, H., Okamura, H., et al. (2001) Clinical Value of Thymidine Kinase in Patients with Cervical Carcinoma. Oncology, 61, 47-54. http://www.ncbi.nlm.nih.gov/pubmed/11474248 https://doi.org/10.1159/000055352

[7] Svobodova, S., Topolcan, O., Holubec, L., Treska, V., Sutnar, A., Rupert, K., et al. (2007) Prognostic Importance of Thymidine Kinase in Colorectal and Breast Cancer. Anticancer Research, 27, 1907-1909. http://www.ncbi.nlm.nih.gov/pubmed/17649793

[8] Luo, P., He, E., Eriksson, S., Zhou, J., Hu, G., Zhang, J., et al. (2009) Thymidine Kinase Activity in Serum of Renal Cell Carcinoma Patients Is a Useful Prognostic Marker. European Journal of Cancer Prevention, 18, 220-224.

http://content.wkhealth.com/linkback/openurl?sid=WKPTLP:landingpage $\& a n=000$ 08469-200906000-00009 https://doi.org/10.1097/CEJ.0b013e328329d817

[9] Zhang, J., Jia, Q., Zou, S., Zhang, P., Zhang, X., Skog, S., et al. (2006) Thymidine Kinase 1: A Proliferation Marker for Determining Prognosis and Monitoring the Surgical Outcome of Primary Bladder Carcinoma Patients. Oncology Reports, 15, 455-461. http://www.spandidos-publications.com/or/15/2/455/abstract https://doi.org/10.3892/or.15.2.455 
[10] Gronowitz, J.S., Hagberg, H., Källander, C.F. and Simonsson, B. (1983) The Use of Serum Deoxythymidine Kinase as a Prognostic Marker, and in the Monitoring of Patients with Non-Hodgkin's Lymphoma. British Journal of Cancer, 47, 487-495.

http://www.pubmedcentral.nih.gov/articlerender.fcgi?artid=2011337\&tool=pmcent rez\&rendertype $=$ abstract

https://doi.org/10.1038/bjc.1983.78

[11] Alegre, M.M., Weyant, M.J.M.J., Bennet, D.T., Yu, J.A., Ramsden, M.K., Elnaggar, A., et al. (2014) Serum Detection of Thymidine Kinase 1 as a Means of Early Detection of Lung Cancer. Anticancer Research, 34, 2145-2151.

http://ar.iiarjournals.org/content/34/5/2145.short

[12] Weagel, E.G., Burrup, W., Kovtun, R., Velazquez, E.J., Felsted, A.M., Townsend, M.H., et al. (2018) Membrane Expression of Thymidine Kinase 1 and Potential Clinical Relevance in Lung, Breast, and Colorectal Malignancies. Cancer Cell International. 